\title{
Le rôle des SIM dans les politiques de sécurité alimentaire : l'expérience du Mali
}

\section{Nango Dembélé}

Ministre, Commissaire

à la sécurité alimentaire du Mali ${ }^{1}$

\section{Salifou B. Diarra}

Directeur de I'Office des produits agricoles du Mali (OPAM) ${ }^{2}$

${ }^{1}$ Commissariat à la sécurité alimentaire (CSA)

Hamdallaye

ACl 2000

Rue 314

Porte 204

Bamako

Mali

$<$ dembele@msu.edu>

${ }^{2}$ Office des produits agricoles du Mali (OPAM)

BP 132

Bamako

Mali

<Salifb.diarra@gmail.com> ne des mesures d'accompagnement de la libéralisation de la commercialisation des céréales au Mali a été la mise en place en 1989 d'un système d'information de marché (SIM) qui devait assurer la transparence et la régulation du marché céréalier à travers la mise à disposition d'informations fiables à tous les acteurs du marché.

En 1989, au terme de quatre semaines de diffusion initiale par le SIM des prix sur les marchés du district de Bamako, une évaluation interne a permis de relever une réduction drastique des différentiels de prix entre les marchés de la périphérie et les marchés de gros qui les approvisionnent. Les gains des consommateurs ont été de 15 à 20 francs $\mathrm{CFA}^{1}$ par kilo de mil, de sorgho ou de maïs acheté, ce qui représentait une baisse de prix d'environ $25 \%$. Étant donné les quantités de céréales échangées à
Bamako, cela a généré une économie de plusieurs centaines de millions de francs CFA. Par ailleurs, les marchés qui étaient souvent cités comme les moins chers ont enregistré plus de fréquentation au détriment des autres marchés.

Aujourd'hui, l'Observatoire du marché agricole (OMA) qui est né de la restructuration du SIM est au centre du dispositif national de sécurité alimentaire (DNSA) du Mali. Ainsi, les recommandations de l'OMA permettent à l'Office des produits agricoles du Mali (OPAM), un autre maillon du DNSA, d'intervenir à des dates pertinentes pour ses achats et ventes dans le cadre des rotations techniques du stock national de sécurité (SNS). Enfin, les autres produits agricoles sont rentrés dans le portefeuille de l'OMA, ce qui traduit le fait que la sécurité alimentaire ne se limite pas aux seules céréales.

\footnotetext{
655,96 francs $\mathrm{CFA}=1$ euro.
}

Tirés à part : N. Dembélé doi: 10.1684/agr.2014.0719
Pour citer cet article : Dembélé N, Diarra SB, 2014. Le rôle des SIM dans les politiques de sécurité alimentaire : I'expérience du Mali. Cah Agric 23 : 231. doi : 10.1684/agr.2014.0719 